\title{
First identified case of VIM-producing carbapenem- resistant Klebsiella pneumoniae in the Republic of Ireland associated with fatal outcome
}

\author{
A R Prior (annarosep@yahoo.com) ${ }^{1}$, C Roche ${ }^{2}$, M Lynch ${ }^{1}$, S Kelly ${ }^{1}, K$ O’Rourke 3 , B Crowley ${ }^{2}$ \\ 1. Department of Clinical Microbiology, Mater Hospital, Dublin, Ireland \\ 2. Department of Microbiology, St James's Hospital, Dublin, Ireland \\ 3. Department of Neurology, Mater Hospital, Dublin, Ireland
}

Citation style for this article:

Prior AR, Roche C, Lynch M, Kelly S, O'Rourke K, Crowley B. First identified case of VIM-producing carbapenem-resistant Klebsiella pneumoniae in the Republic of Ireland associated with fatal outcome. Euro Surveill. 2010;15(50):pii=19752. Available online: http://www.eurosurveillance.org/ViewArticle.aspx?Articleld=19752

Article published on 16 December 2010

To the editor: Following the recent review of carbapenem-resistant Enterobacteriaceae (CRE) in Europe [1], we would like to add that a first case of VIM-1 producing carbapenem-resistant Klebsiella pneumoniae has now also occurred in Ireland, associated with the repatriation of a patient from a Greek hospital.

In September 2010 a woman in her mid-fifties, was transferred from a Greek hospital to the intensive care unit of our institution, with severe herpes simplex encephalitis. She was empirically treated with vancomycin and meropenem for nosocomial pneumonia. Pseudomonas aeruginosa and Enterobacter cloacae cultured from respiratory specimens were susceptible to meropenem. However, $K$. pneumoniae resistant to meropenem was identified from a swab collected from a deep sacral pressure sore. The patient was immediately isolated. Treatment with tigecycline and intravenous colistin was added, but the patient died within seven days of her transfer. No isolates of CRE were detected in samples collected from patient's contacts.

The patient's isolate was found to be resistant to all beta-lactam antibiotics, including the carbapenems, as well as all aminoglycosides and fluoroquinolones, but remained susceptible to colistin and had intermediate susceptibility to tigecycline $(2 \mathrm{mg} / \mathrm{L})$ according to EUCAST criteria [2]. Carbapenemase production was indicated by a positive modified Hodge plate test. Phenotypic screening for $K$. pneumoniae carbapenemase production was negative, but positive for production of a metallo-beta-lactamase. The presence of the gene encoding VIM-1 was confirmed by sequence analysis (GenBank accession number HQ442296).

This first VIM-1-producing $K$. pneumoniae isolate in Ireland belonged to the group of enterobacteria producing the recently reported New Delhi metallo-betalactamase NDM-1. To date, there is only one report of endemic class A KPC-2 production in $K$. pneumoniae in Ireland [3].
The case highlights the importance of prompt implementation of infection control measures in patients repatriated from countries where CREs are endemic. Such patients should be placed in isolation using contact precautions until results of surveillance cultures are available [4].

References

1. Grundmann H, Livermore D, Giske C, Canton R, Rossolini G, Campos J, et al. Carbapenem-non-susceptible Enterobacteriaceae in Europe: conclusions from a meeting of national experts. Euro Surveill. 2010;15(46):pii=19711. Available from: http://www.eurosurveillance.org/ViewArticle. aspx?Articleld $=19711$

2. European Committee on Antimicrobial Susceptibility Testing (EUCAST). EUCAST Procedure for Harmonising and Defining Breakpoints. The Swedish Reference Group for Antibiotics. [Accessed:10/10/2010]. Available from: http://www.srga.org/ eucastwt/bpsetting.htm

3. Roche C, Cotter M, O'Connell N, Crowley B. First identification of Class A carbapenemase-producing Klebsiella pneumoniae in the Republic of Ireland. Eurosurveill. 2009;14(13):pii=19163. Available from: http://www.eurosurveillance.org/ViewArticle. aspx?Articleld $=19163$

4. Centers for Disease Control and Prevention (CDC). Guidance for control of infections with carbapenem-resistant or carbapenemase-producing Enterobacteriaceae in acute care facilities. MMWR Morb Mortal Wkly Rep. 2009; 58(10):256-60. 